\title{
Issues of Measurability on the Transparency of the Administrative Procedure Among Clients
}

\section{Balázs Hohmann}

Dr., Assistant lecturer, University of Pécs, Faculty of Law, Department of Administrative Law

\begin{abstract}
The requirement for the transparency of the administrative procedure and the expectations for this arise step by step during the relevant legislation of European countries. However, the concept of this often used adjective remains vague in many cases, making it difficult to put it into practice. However, the requirement is of great importance for modern public administration systems in the 21st century: transparency has been proven to play a major role in building and maintaining citizens' trust in public administration and public bodies. Therefore, in addition to the objective meaning of transparency, which can be explored from legislation and the relevant legislative document, it is important to use empirical methods to explore the perceptions of transparency in clients. Transparency of the administrative procedure is in many respects a subjective concept. After all, in addition to the legislation on the administrative procedure and the consistent application process, as well as their communication, it depends to a large extent on the perceptions, knowledge, information and experience of the main stakeholders, usually natural persons clients. The presentation and the related study aim to explore this sense of transparency with empirical methods and seek to make practical findings that are independent of national specificities.
\end{abstract}

Keywords: administrative procedure, client, transparency, empirical methods. 